\title{
Efeito da desalcoolização parcial de vinhos tintos finos através da liofilização
}

\author{
João Felippeto ${ }^{1}$, Francieli Artismo² e Alberto Fontanella Brighenti ${ }^{3}$
}

\begin{abstract}
Resumo-Bebidas com teor alcoólico reduzido têm ganhado espaço no mercado consumidor mundial. Assim, o desenvolvimento de tecnologias conservadoras, capazes de remover parcialmente o álcool sem prejudicar as suas características sensoriais, é de grande interesse às indústrias vinícolas. O objetivo deste trabalho foi testar a eficiência e compreender os efeitos da liofilização para a desalcoolização parcial de vinhos. As amostras foram submetidas ao processo de liofilização e as cinéticas de decaimento ou concentração dos componentes da matriz do vinho foram sequencialmente avaliadas, através da correlação entre os valores dos parâmetros físico-químicos e o tempo de exposição, em intervalos entre 30 minutos e 120 minutos. O método se mostrou eficiente para a redução dos compostos voláteis do vinho, com destaque para o álcool $\left(0,038^{\circ} \mathrm{GL}\right)$ e para a água $(0,9 \mathrm{~mL})$, por minuto. Em função da redução gradual do volume, foram observados aumentos na acidez $\left(0,092 \mathrm{mEq} \mathrm{L}^{-1}\right)$ e nos índices de polifenóis totais $\left(2,77 \mathrm{mgEAG}^{-1}\right)$ por minuto. Relativamente aos aspectos sensoriais, foram verificados decréscimos com maior variabilidade nos descritores associados com a intensidade olfativa e com a nitidez gustativa.
\end{abstract}

Termos de indexação: teor alcoólico reduzido; bebidas alcoólicas; qualidade sensorial.

\section{Effect of partial dealcoholization of fine red wines through lyophilization}

Abstract - Drinks with reduced alcohol content have gained space in the world consumer market. Thus, the development of conservative technologies, capable of partially removing alcohol without impairing its sensorial characteristics, is of great interest to the wine industry. The objective of this study was to test the efficiency and to understand the effects of the lyophilization process for partial dealcoholization of wines. The samples were submitted to the lyophilization process, and the kinetics of decay or concentration of the wine matrix components were evaluated by correlation between the values of the physicochemical parameters and the time of exposure, in intervals from 30 to 120 minutes. The method was efficient to reduce the volatile compounds of wine, with emphasis on alcohol $\left(0.038^{\circ} \mathrm{GL}\right)$ and water $(0.9 \mathrm{~mL})$ per minute. As a result of the gradual volume reduction, increases in acidity $\left(0.092 \mathrm{mEqL}^{-1}\right)$ and total polyphenol indices $\left(2.77 \mathrm{mg} \mathrm{EAGL}^{-1}\right)$ were observed per minute. Regarding sensorial aspects, there were decreases with greater variability in the descriptors associated with olfactory intensity and taste clarity.

Index terms: reduced alcohol content; alcoholic beverages; sensory quality.

\section{Introdução}

O consumo de vinhos com teor alcoólico reduzido tem aumentado em diversos países. De acordo com as agências Euromonitor International (WINE, 2012) e Institute of Wine and Spirit Record (2012), o Reino Unido representa o maior mercado mundial de vinhos, com valores próximos a $£ 14$ bilhões em 2011. Nesses países, os vinhos com menor teor alcoólico tiveram a sua produção aumentada em $26 \%$, com uma valorização de preços próxima aos 39\% entre março de 2011 a março de 2012
(BRUWER et al., 2014; WINE INTELLIGENCE, 2012). Por ser altamente competitivo, o ambiente de mercado do Reino Unido pode fornecer ideias claras sobre as modificações no comportamento do consumidor mundial, bem como subsidiar importantes estratégias de vendas no varejo (BRUWER et al., 2011, 2012).

A produção e a comercialização de vinhos com reduzido teor alcoólico são uma excelente oportunidade mercadológica em todo o mundo. Além disso, nos últimos anos, o mercado mundial de alimentos e bebidas tem observa- do uma forte modificação no padrão de consumo: a do produto saudável e conveniente. Esta mudança no comportamento do mercado está relacionada à busca de produtos alternativos capazes de oferecer menos calorias e, no caso das bebidas fermentadas, uma redução dos efeitos do álcool. Neste viés, as bebidas fermentadas com baixo teor alcoólico têm sido comercializadas como opção para os consumidores e parecem representar uma completa modificação no padrão tradicional de consumo, situação comparável aos produtos light e diet produzidos pela indústria de la-

Recebido em 22/8/2018. Aceito para publicação em 20/11/2018.

http://dx.doi.org/10.22491/RAC.2019.v32n2.12

1 Enólogo, Msc., Epagri / Estação Experimental de São Joaquim, rua João Araújo Lima, 102, bairro Jardim Caiçara, $88600-000$ São Joaquim, SC, e-mail: joaofelippeto@epagri.sc.gov.br.

${ }^{2}$ Engenheira-agrônoma / Universidade Estadual de Santa Catarina, Av. Luiz de Camões, 2090, bairro Conta Dinheiro, Lages, SC, e-mail: francieli_artismo@ hotmail.com

${ }^{3}$ Eng. agrônomo, Dr., Epagri / Estação Exp. de São Joaquim, rua João Araújo Lima, 102, bairro Jardim Caiçara, 88600-000 São Joaquim, SC, e-mail: albertobrighenti@epagri.sc.gov.br 
ticínios (SILVA, 2004; ORDUÑA, 2010; GAMBUTI et al., 2011).

Diversas tecnologias têm sido desenvolvidas para a redução do etanol em vinhos na fase pós-fermentativa: destilação por coluna (cone spinning) e extração supercrítica de $\mathrm{CO}_{2}$ combinada com destilação a vácuo (SEIDLITZ et al., 1991); osmose reversa (PILIPOVIK \& RIVEROL, 2005); separação por membrana (LABANDA et al., 2009); pervaporação (TAKÁCS et al., 2007); destilação osmótica (LIGUORI et al., 2013). Estas metodologias, no entanto, apresentam alto custo e afetam os atributos sensoriais do vinho. Nesse contexto, compreende-se a importância do desenvolvimento ou da validação de métodos capazes de gerar processos industriais eficientes para reduzir o teor alcoólico nos vinhos sem prejuízo às suas características olfato-gustativas.

A liofilização pode ser um método adequado para a desalcoolização parcial de vinhos considerando a natureza conservadora desta técnica que, segundo Marques \& Costa (2015), produz alterações mínimas nos atributos de natureza nutricional e sensorial dos alimentos por permitir a retenção de cerca de $80 \%$ das substâncias voláteis e aromas. Esse método utiliza temperaturas mais baixas que o ponto de congelamento da água e pressão menor do que a atmosférica para promover a sublimação da água desde o centro do gelo com mínimo deslocamento de sólidos solúveis para a superfície. Além disso, as reações oxidativas e não oxidativas são mínimas, já que que permite manutenção das paredes celulares (BOSS, 2007; MARQUES, 2008; VAN CLEEF et al., 2010; MARQUES $\&$ COSTA, 2015). Considerando a escassez de informações sobre o comportamento dos vinhos frente a esse processo, o objetivo desta pesquisa foi avaliar a eficiência e compreender os efeitos da liofilização sobre os parâmetros físicoquímicos e sensoriais de vinhos tintos finos.

\section{Material e métodos}

O trabalho foi realizado no Laboratório de Enoquímica da Epagri - Estação Experimental de São Joaquim, SC. $\mathrm{O}$ vinho utilizado foi elaborado a partir das variedades Cabernet Sauvignon $(70 \%)$ e Merlot (30\%), cultivadas na região de São Joaquim durante o ciclo de $2010 / 2011$. As amostras contendo exatamente $500 \mathrm{~mL}$ de vinho a $20^{\circ} \mathrm{C}$ foram acondicionadas em tubos de liofilização com capacidade para $1000 \mathrm{~mL}$ e resfriadas até o ponto de congelamento através de uma solução hidroalcoólica a $-35^{\circ} \mathrm{C}$. Após o congelamento dos vinhos, os tubos foram acoplados a um liofilizador EYELA (Tokyo Rikakikai Freezer FD) e imediatamente expostos a uma pressão de $12 \mathrm{~Pa}$. As amostras foram retiradas em intervalos lineares de tempo: $0 \mathrm{~min}$. (testemunha), $30 \mathrm{~min} ., 60 \mathrm{~min} ., 90 \mathrm{~min}$. e $120 \mathrm{~min}$. Os intervalos foram estabelecidos a partir de estudos prévios sobre a velocidade de redução dos componentes voláteis do vinho, realizados no mesmo equipamento.

Como forma de compreender a performance do vinho frente aos tratamentos, foram estudadas as cinéticas de decaimento dos componentes voláteis, bem como a concentração dos componentes fixos presentes na matriz do vinho. Tais cinéticas foram estabelecidas através da correlação entre os valores dos parâmetros físico-químicos e o tempo de exposição ao processo de liofilização. Todos os tratamentos foram em triplicata, perfazendo quinze amostras. Os parâmetros físico-químicos estudados foram: acidez total titulável $\left(\mathrm{mEq} \mathrm{L} \mathrm{L}^{-1}\right), \mathrm{pH}$, teor alcoólico $\left({ }^{\circ} \mathrm{GL}\right)$ e densidade relativa, conforme metodologia descrita por Rizzon (2010). O índice de polifenóis totais (IPT) foi determinado de acordo com Singleton \& Rossi (1965), utilizando o reagente Folin-Ciocalteu. As concentrações de polifenóis totais foram lidas em espectrofotômetro de UV/VIS na faixa espectral de 760nm, quantificadas por meio de uma curva de calibração com ácido gálico e expressas em miligramas de Equivalente de Ácido Gálico por litro (mgEAG L-1). A densidade ótica (DO) também foi determinada através da espectrofotometria, nos comprimentos de onda de $420 \mathrm{~nm}$, $520 \mathrm{~nm}$ e $620 \mathrm{~nm}$ (medidos pela absorbância), em cubetas de $1,00 \mathrm{~mm}$ de percurso ótico. Foram calculados os índices de cor (IC $=A 420+A 620+A 320)$ e os teores de cor $(T=A 420 / A 520)$, de acordo os métodos propostos por Glories (1984) e Amerine \& Ough (1988).
As análises sensoriais foram realizadas através de um painel composto por cinco julgadores previamente treinados, utilizando fichas de provas padrão da Organização Internacional do Vinho (OIV, 1990). Quinze minutos antes das análises, os vinhos foram distribuídos em taças tipo ISO previamente codificadas. Cada amostra foi avaliada quanto aos seus aspectos visuais, olfativos, gustativos e apreciação global. A ordenação dos dados foi feita com o auxílio de planilhas Excel, sendo calculadas, para cada variável, as médias e os desviospadrão amostrais. Com os dados obtidos, foram estabelecidas as correlações e elaborados os gráficos que demonstram as dinâmicas de decaimento ou a concentração dos diversos componentes do vinho submetido ao método em avaliação.

\section{Resultados e discussão}

O teor alcoólico das amostras, que inicialmente era de $12^{\circ} \mathrm{GL}$, sofreu uma diminuição de aproximadamente $0,038^{\circ} \mathrm{GL}$ por minuto durante a exposição do vinho ao processo de liofilização, chegando a $7,3^{\circ} \mathrm{GL}$ no final de 120 minutos (Figura 1A). Liguori et al. (2013), utilizando o método da destilação osmótica para a redução do teor de etanol em vinho tinto da variedade Aglianico com um teor alcoólico inicial de $13,0^{\circ} \mathrm{GL}$, obtiveram reduções que chegaram a $9,9^{\circ} \mathrm{GL}$ nos mesmos 120 minutos, o que demonstra que a liofilização proporciona, comparativamente, um menor fluxo de extração do álcool. A volatilização do álcool verificada nas condições do experimento pode ser explicada pelo princípio físico da liofilização, de forma semeIhante ao que ocorre com a água durante a sublimação. Neste processo, a evaporação da água ocorre diretamente do estado sólido para o gasoso, desde que a pressão de vapor e a temperatura da camada de gelo se mantenham abaixo do ponto tríplice da água que é $0,0099^{\circ} \mathrm{C}$ a uma pressão atmosférica menor do que 610Pa (ORDÓÑES, 2005; MARQUES \& COSTA, 2015). Por outro lado, assim como a água, os demais componentes de natureza volátil também são evaporados, na interface entre o gelo e o ar, através do fluxo causado pela baixa 

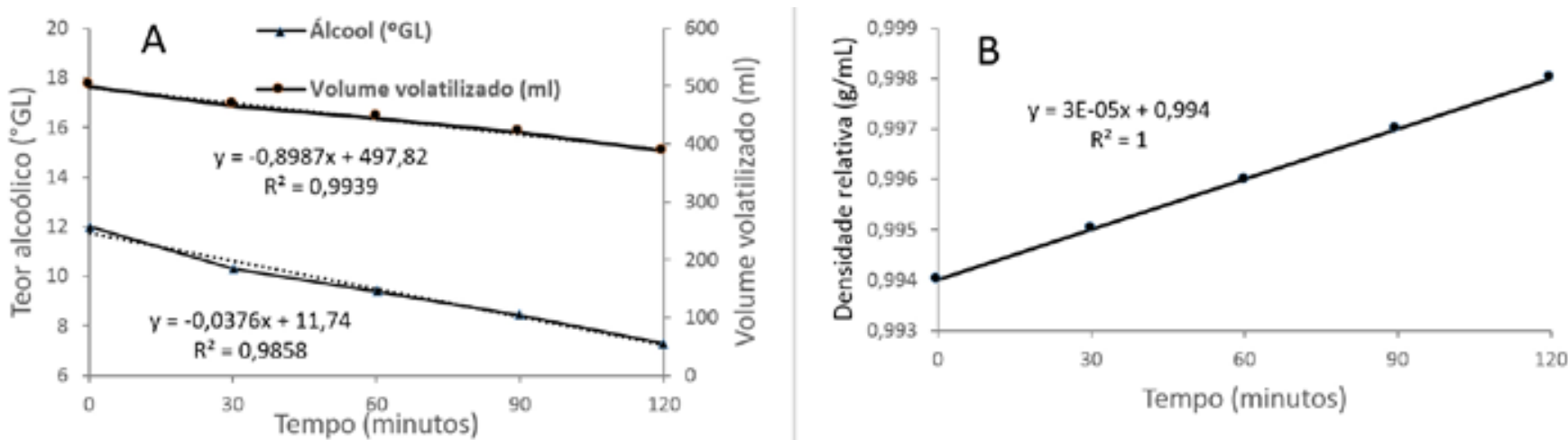

Figura 1. Teor alcoólico ( $\left.{ }^{\circ} \mathrm{GL}\right)$, volume volatilizado $(\mathrm{mL})(\mathrm{A})$ e densidade relativa $(\mathrm{g} / \mathrm{mL})(B)$ dos vinhos submetidos ao processo de desalcoolização parcial através do método da liofilização

Figure 1. Alcohol content ( $\left.{ }^{\circ} \mathrm{GL}\right)$, volatilized volume $(\mathrm{ml})(\mathrm{A})$ and density of wines $(B)$ submitted to the partial de-alcoholization process by the freeze-drying method

pressão atmosférica, consequentemente reduzindo os seus teores. Observase uma tendência com alta linearidade no decaimento deste parâmetro, o que demonstra a eficiência do método utilizado como promotor de extração dos componentes voláteis, com destaque para os álcoois e para a água. Também nota-se uma redução linear no volume das amostras, a uma taxa de, aproximadamente, $0,9 \mathrm{~mL}$ por minuto, reduzindo de $500 \mathrm{~mL}$ para $388 \mathrm{~mL}$ no final do processo. O principal efeito da extração dos principais solventes da matriz do vinho (álcool e água) é o aumento da densidade relativa das amostras, o que pode ser observado na Figura 1B. Este parâmetro teve os seus valores aumentados de forma linear a uma taxa de aproximadamente 0,001 pontos a cada 30 minutos. A elevação numérica da densidade se deve à concentração de moléculas com menor volatilidade, como os polifenóis, além dos sólidos dissolvidos na matriz complexa da mistura. Estudos de métodos para redução do etanol de vinhos através da nanofiltração também mostraram aumentos nas quantidades relativas de compostos moduladores da densidade como o açúcar, ácido total, extrato total e extrato sem açúcar, em função da extração da água e do álcool (BANVOLGYI et al., 2006). É importante referir que a expectativa dos consumidores dos vinhos tintos elaborados nas altas altitudes é a de uma bebida com uma estrutura mais densa. Nesse sentido, a liofilização pode contribuir positivamente para o atendimento dessa expectativa.
No que se refere à acidez total titulável (ATT), os valores aumentaram a uma taxa de aproximadamente $2,76 \mathrm{mEq} \mathrm{L}^{-1}$ a cada 30 minutos, modificando o seu teor relativo de $53,08 \mathrm{mEq} \mathrm{L}^{-1}$ para $64,12 \mathrm{mEq} \mathrm{L}^{-1}$ após o transcurso de 120 minutos (Figura 2). Os vinhos contêm uma matriz ácida composta por diversos ácidos orgânicos, com destaque para o tartárico, málico e cítrico, que são derivados da própria uva, e aqueles provenientes do metabolismo microbiológico durante o processo fermentativo, como o succínico, acético e lático (BENASSI, 1997). Rolle et al. (2018), utilizando a técnica da destilação osmótica para a redução do álcool nos vinhos, verificaram aumentos na concentração dos ácidos tartárico, lático, succínico e cítrico, mesmo em pequenas reduções no teor alcoólico $\left(0,2^{\circ} \mathrm{GL}\right.$ a $\left.2^{\circ} \mathrm{GL}\right)$, sem no entanto, ocasionar modificações significativas na ATT. Estes dados também estão de acordo com Liguori et al. (2013). A constatação do aumento da ATT na matriz do vinho pode ser explicada pela redução dos solventes (principalmente a água), e pela consequente concentração dos ácidos totais. Esta observação pode ser ratificada pelo comportamento do $\mathrm{pH}$ que teve os seus valores reduzidos de 3,85 para 3,81 , durante $\mathrm{o}$ transcurso de 120 minutos (Figura 2). $\mathrm{O}$ pH corresponde à concentração de hidrogênio iônico proveniente dos ácidos orgânicos, além da concentração de potássio, e representa a acidez real do vinho (GABAS et al, 1994; RIZZON \&

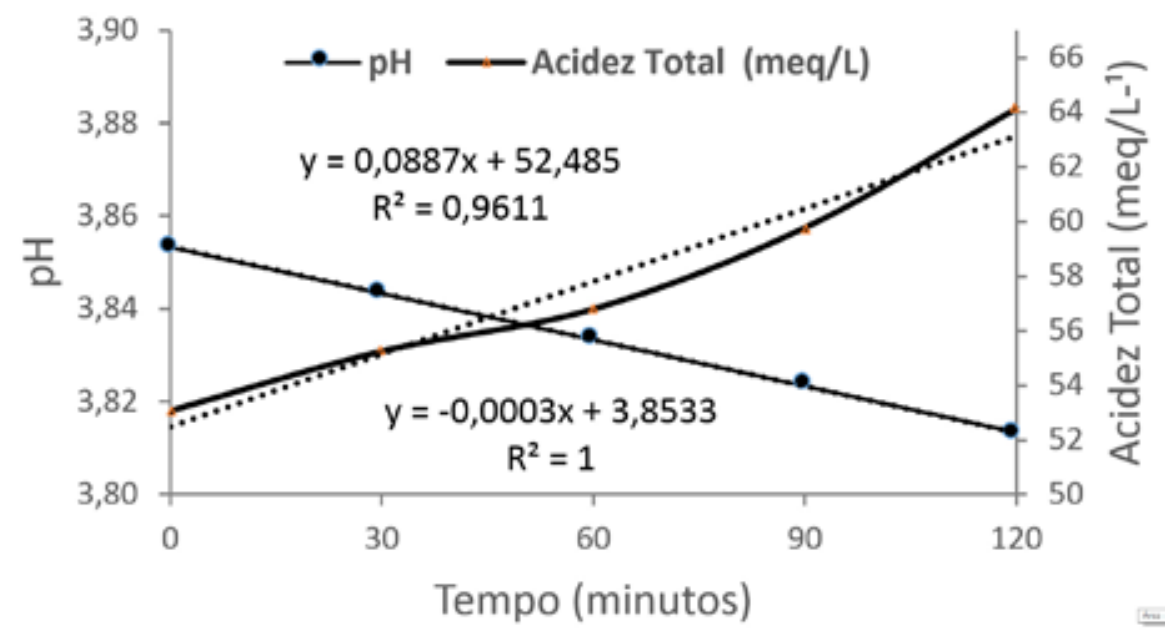

Figura 2. $\mathrm{pH}$ e acidez total (meqL $\mathrm{L}^{-1}$ ) dos vinhos submetidos ao processo de desalcoolização parcial através do método da liofilização

Figure 2. $\mathrm{pH}$ and total acidity (meqL $\mathrm{L}^{-1}$ ) of wines submitted to the partial de-alcoholization process by the freeze-drying method 
MIELE, 2002). Esses resultados estão de acordo com Lança (2011), que estudou o comportamento de vinhos brancos e tintos parcialmente desalcoolizados através dos métodos da osmose inversa e da nanofiltração, quando foi constatado uma ligeira redução do $\mathrm{pH}$ nos tintos, ocasionada pelo aumento da acidez total. É importante referir que a elevação dos valores da ATT nos vinhos pode ser benéfica para a sua qualidade, especialmente nos brancos elaborados com uvas sobrematuradas ou provenientes de regiões de clima quente, onde os ácidos orgânicos são fortemente degradados durante o período da maturação. Quanto aos polifenóis totais (Figura 3A), é possível observar uma variação de, aproximadamente, $20,3 \%$, aumentando de 1633,45 para 1965,73 mgEAG L $^{-1} \mathrm{em}$ 120 minutos. Embora o índice de polifenóis totais represente a totalidade dos compostos fenólicos presentes num determinado vinho, sem distinção das frações relativas de cada grupo, os resultados obtidos demonstram que estas substâncias não são retiradas do vinho através do fluxo promovido pela liofilização. Portanto, o aumento do IPT pode ser explicado pela baixa capacidade de volatilização dos polifenóis e da sua consequente concentração nos vinhos, quando submetidos a esse método. Essa constatação está de acordo com os resultados obtidos por Liguori et al. (2010) e Belisario-Sanchez et al. (2009), utilizando a técnica da osmose direta. É importante referir que os polifenóis são substâncias que interferem fortemente nas características cromáticas dos vinhos, o que foi demonstrado através das análises espectrofotométricas da cor das amostras.

As características da cor, identificadas através do índice de cor (IC) e da tonalidade de cor (TC), estão demonstrados na figura 3B. Houve aumento de aproximadamente $28 \%$ no IC nas condições deste experimento. De acordo com Glories (1984), o IC é definido como a soma das densidades óticas medidas nas faixas espectrais em que: A420 (amarelo) representa a proporção de taninos; A520 (vermelho), as antocianinas; e A620 (violeta-azul), as condensações entre as catequinas e as antocianinas. Portanto, o aumento do IC ratifica os dados obtidos pela quantificação do IPT (Figura 3A), considerando que uma parte importante da estrutura polifenólica é responsável pela cor. Entretanto, o teor de cor, que é definido pela razão entre as faixas espectrofotométricas (A420/A520), permaneceu praticamente inalterado (Figura 3B). Os fatores que podem modificar a tonalidade são as reações de condensação de antocianinas e taninos envolvendo etanal (aumento dos valores de A520); reações de oxidação envolvendo a degradação das antocianinas (redução de A520 e A620); polimerização de taninos, tornando-se castanhos (aumento do A420); oxidação das combinações taninos-antocianas evoluindo naturalmente para o laranja (aumento do A420 e diminuição do A620) (GLORIES, 1984). Neste sentido, os vinhos oxidados ou excessivamente envelhecidos tendem a ser menos vermelhos, evoluindo para uma cor mais alaranjada ou telha. A linearidade dos valores do teor de cor observada neste trabalho mostra que não houve fortes modificações nas relações oxidativas ou poliméricas entre os principais compostos fenólicos e, portanto, o método pode ser considerado pouco agressivo ou mesmo conservador para a estrutura polifenólica total.

Quanto às análises sensoriais do vinho, não foram observadas grandes modificações nos descritores responsáveis pelas características visuais, o que pode ser comprovado através dos baixos valores dos coeficientes de variação da limpidez e do aspecto (CV\% menor do que 6\%) (Tabela 1). Essa constatação evidencia que não houve precipitações ou turvações dos sólidos dissolvidos na matriz do vinho ocasionadas pelo método em estudo. Por outro lado, a intensidade visual teve os seus valores reduzi- -
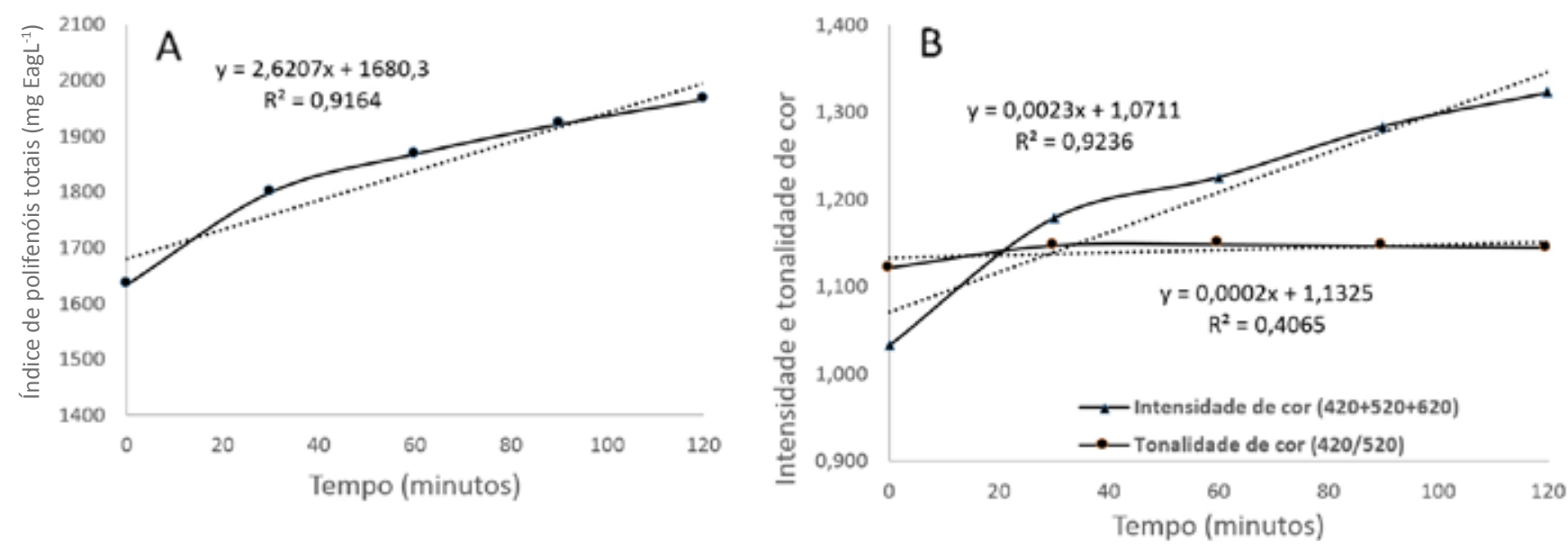

Figura 3. Índice de polifenóis totais $\left(\mathrm{mgEAGL}^{-1}\right)(\mathrm{A})$ e intensidade de cor dos vinhos (B) submetidos ao processo de desalcoolização parcial através do método da liofilização.

Figure 3. Index of total polyphenols $\left(m g E A G L^{-1}\right)(A)$ and color intensity of wines $(B)$ submitted to the partial de-alcoholization process by the freeze-drying method 
Tabela 1. Descritores sensoriais percentuais do vinhos submetido à desalcoolização parcial através do método da liofilização

Table 1. Sensory descriptors of wines subjected to partial de-alcoholization by the freezedrying method

\begin{tabular}{|c|c|c|c|c|c|c|c|}
\hline & \multirow[b]{2}{*}{ Descritores } & \multicolumn{5}{|c|}{ Minutos } & \multirow[b]{2}{*}{$\mathrm{CV}(\%)^{1}$} \\
\hline & & 0 & 30 & 60 & 90 & 120 & \\
\hline \multirow{3}{*}{ Visual } & Limpidez & 100,0 & 100,0 & 92,0 & 92,0 & 92,0 & 4,6 \\
\hline & Aspecto & 96,0 & 95,0 & 88,0 & 88,0 & 84,0 & 5,7 \\
\hline & Intensidade & 85,0 & 71,9 & 62,5 & 62,5 & 62,5 & 13,3 \\
\hline \multirow{3}{*}{ Olfativo } & Nitidez & 80,0 & 66,7 & 56,7 & 56,7 & 53,3 & 16,6 \\
\hline & Qualidade & 87,5 & 75,0 & 67,5 & 67,5 & 65,0 & 12,2 \\
\hline & Intensidade & 80,0 & 68,8 & 65,0 & 55,0 & 40,0 & 24,5 \\
\hline \multirow{4}{*}{ Gustativo } & Nitidez & 76,7 & 62,5 & 53,3 & 53,3 & 46,7 & 18,8 \\
\hline & Qualidade & 78,2 & 69,3 & 56,4 & 56,4 & 53,6 & 15,4 \\
\hline & Persistência & 85,0 & 68,8 & 65,0 & 65,0 & 62,5 & 12,5 \\
\hline & Global & 87,3 & 81,8 & 70,9 & 70,9 & 67,3 & 10,5 \\
\hline
\end{tabular}

(1) Coeficiente de variação(\%)

dos ao longo do processo, considerando o valor do seu $\mathrm{CV} \%$, que chegou $13,3 \%$. Paradoxalmente, os valores da intensidade visual obtidos nas degustações e os lidos através da espectrofotometria são divergentes. Embora as causas desta diferença não estejam completamente elucidadas, é preciso considerar a natureza das análises. Se por um lado as leituras obtidas eletronicamente informam as variações de cor em três frequências restritas: amarelo, vermelho e azul-violeta, por outro, as sensoriais informam as características globais do vinho, considerando todo o espectro visível através do olho humano, porém com critérios subjetivos e sujeitos a interferências não matemáticas. Quanto às características olfativas, ficou evidenciado que os aromas são influenciados pela liofilização de forma que, quanto maior o tempo de exposição ao método, menores são as suas percepções. As maiores variações foram verificadas no descritor olfativo responsável pela intensidade (CV 24,5\%) (Tabela 1). Essa redução se deve, provavelmente, à natureza volátil dos compostos químicos responsáveis pelos aromas da bebida. De acordo com Vilanova et al. (2013) os álcoois monoterpênicos são os com- cionadas para a redução do teor alcoólico de vinhos.

\section{Conclusões}

A liofilização é um método eficiente para a redução do teor alcoólico de vinhos tintos por possibilitar a extração do etanol a uma taxa linear constante.

Os compostos com menor volatilidade como os polifenóis, os ácidos orgânicos e os sólidos dissolvidos na matriz complexa da mistura são preservados durante o processo, o que aumenta as suas concentrações relativas.

Os componentes responsáveis pelas percepções sensoriais têm um decréscimo proporcional ao tempo de exposição ao método, tornando-o inapropriado para grandes reduções nos teores alcoólicos dos vinhos.

\section{Referências:}

postos mais importantes do aroma da uva e responsáveis por diversas notas aromáticas nos vinhos. Considerando a natureza volátil desses álcoois monoterpênicos, é possível inferir que uma parte importante destes compostos, inicialmente presentes nas amostras, sofrem uma redução quantitativa através do mesmo fluxo que foi capaz de volatilizar os demais álcoois.

As características gustativas também sofreram modificações durante a exposição ao método, o que pode ser explicado pela gradativa redução dos componentes voláteis do vinho, já que estas substâncias desempenham papeis importantes na percepção gustativa para muito além dos descritores básicos, como doçura, amargor, acidez, adstringência e salinidade. As maiores variabilidades foram observadas nos descritores responsáveis pela nitidez (CV $18,8 \%$ ) e pela qualidade (CV $15,4 \%$ ) (Tabela 1). Diversos autores obtiveram resultados semelhantes (DIBAN et al., 2013; LIGUORI et al., 2010; LIGUORI et al., 2013; MANGINDAAN et al., 2018), evidenciando que a preservação dos componentes aromáticos e gustativos representa um grande desafio para o desenvolvimento de tecnologias dire-
AMERINE, M. A.; OUGH, C. S. Wine and must analysis. New York: Willey, 1988.

BANVOLGYI, S.; KISS, I.; BEKASSY-MOLNAR, E.; VATAI, G. Concentration of red wine by nanofiltration. Desalination, Amsterdam, v. 198, n. 1-3, p. 8-15, jan. 2006.

BELISARIO-SANCHEZ, Y. Y.; TABOADA-RODRÍGUEZ, A; MARIN-INIESTA, F.; LÓPEZ-GÓMEZ, $A$. Dealcoholized wines by spinning cone column distillation: Phenolic compounds and antioxidant activity measured by the 1,1-diphenyl-2-picrylhydrazyl method. Journal of Agricultural and Food Chemistry, Washington, DC, v. 57, n. 15, p.6770-6778, jul. 2009.

BENASSI, M.T. Metodologia analítica para avaliação de parâmetros físico-químicos e sensoriais de qualidade em vinhos Riesling Itálico nacionais. 1997. 164f. Tese (Doutorado em Engenharia de Alimentos) - Universidade Estadual de Campinas, Campinas, 1997.

BOSS, E.A. Modelagem e otimização do processo de liofilização: aplicação para leite desnatado e café solúvel. 2007. 87f. Tese (Doutorado em Engenharia Química) - Universidade Estadual de Campinas, Campinas, 2007.

BRUWER, J.; SALIBA, A. J.; MILLER, B. Con- 
sumer behaviour and sensory preference differences: implications for wine product marketing. Journal of Consumer Marketing, Bingley, v. 28, n. 1, p. 5-18, 2011.

BRUWER, J.; LESSCHAEVE, I.; CAMPBELL, B. L. Consumption dynamics and demographics of Canadian wine consumers: retailing insights from the tasting room channel. Journal of Retailing and Consumer Services, Amsterdam, v. 19, n. 1, p. 45-58, 2012.

BRUWER, J.; JIRANEK, V.; HALSTEAD, L.; SALIBA A. Lower alcohol wines in the UK market: some baseline consumer behaviour metrics. British Food Journal, Bingley, v. 116, n. 7, p. 1143-1161, 2014.

DIBAN, N.; ARRUTI, A.; BARCELÓ, A.; PUXEU, M.; URTIAGA, A.; ORTIZ, I. Membrane dealcoholization of different wine varieties reducing aroma losses. Modeling and experimental validation. Innovative Food Science and Emerging Technologies, Amsterdam, v. 20, p. 259-268, mai. 2013.

GABAS, N.; RATSIMBA, B.; GERBAUD, V. Les sels tartriques dans les vins: solubilité et sursaturation. In: Lallemand (Ed.). La microbiologie des vins mousseux: la stabilisation des vins : mécanismes et évaluation. Toulouse: Lallemand, 1994, p. 95-98.

GAMBUTI, A.; RINALDI, A.; LISANTI, M. T.; PESSINA, R.; MOIO, L. Partial dealcoholisation of red wines by membrane contactor technique: influence on colour, phenolic compounds and saliva precipitation index. European Food Research and Technology, Nova York, v. 233, n. 4, p. 647-655, jul. 2011.

GLORIES, Y. La couleur des vins rouges. 2e partie : mesure, origine et interprétation. Journal International des Sciences de la Vigne et du Vin, França, v. 18, n. 4, p. 253271, 1984.

INSTITUTE of Wine and Spirit Record. London, 2012. Disponível em: https://bit. Iy/2TRxZPg. Acesso em: 30 ago. 2018.

LABANDA, J.; VICHI, S.; LLORENS, J.; LOPEZTAMAMES, E. Membrane separation technology for the reduction of alcoholic degree of a white model wine. LWT - Food Science and Technology, Amsterdam, v. 42, n. 8, p. 1390-1395, mar. 2009.

LANÇA, T.M. Desalcoolização parcial de vinhos por nanofiltração e osmose inversa: efeito do nível de desalcoolização. 2011. 93f. Dissertação (Mestrado em Viticultura e Enologia) - Instituto Superior de Agronomia, Universidade Técnica de Lisboa, Lisboa, 2011.

LIGUORI, L.; ATTANASIO, G.; ALBANESE, D.; DI MATTEO, M. Aglianico wine dealcoholization tests. In: EUROPEAN SYMPOSIUM ON COMPUTER AIDEDPROCESS ENGINEERING, 20., 2010, Ischia. Anais [...]. Ischia: Escape, 2010, 325p.

LIGUORI, L.; RUSSO, P.; ALBANESE, D.; DI MATTEO, M. Evolution of quality parameters during red wine dealcoholization by osmotic distillation. Food Chemistry, Amsterdam, v. 140, n. 1-2, p. 68-75, feb. 2013.

MANGINDAAN D.; KHOIRUDDINB, K.; WENTENB, I.G. Beverage dealcoholization processes: past, present, and future. Trends in Food Science \& Technology, Amsterdam, v. 71, p. 36-45, 2018.

MARQUES, E.C.; COSTA, S.R.R. Estudo da liofilização pela engenharia de produto no processamento industrial de alimentos. Acta Tecnológica, São Luís, v. 10, n. 1, p. 44-52, 2015.

MARQUES, L.G. Liofilização de frutas tropicais. 2008. 249f. Tese (Doutorado em Engenharia Química) - Universidade Federal de São Carlos, São Carlos, 2008.

OIV. OIV standard for international wine competitions and spirituous beverages of vitivinicultural origin. Paris: OIV, 2009. 19p.

ORDÓÑES, J.A. Tecnologia de alimentos: componentes dos alimentos e processos. Porto Alegre: Artmed, 2005. 294p. 1 v.

ORDUÑA, R.M. Climate change associated effects on grape and wine quality and production. Food Research International, Amsterdam, v. 43, n. 7, p. 1844-1855, mai. 2010.

PILIPOVIK, M.V.; RIVEROL, C. Assessing dealcoholization system based on reverse osmosis. Journal Food Engineering, Amsterdam, v. 69, n. 4, p. 437-441, ago. 2005.

RIZZON, L.A.; MIELE, A. Acidez na vinificação em tinto das uvas Isabel, Cabernet Sauvignon e Cabernet Franc. Ciência Rural, Santa Maria, v. 32, n. 3, p. 511-515, 2002.

RIZZON, L.A. (Ed.). Metodologia para análise de vinho. Brasília, DF: Embrapa, 2010. 120p.
ROLLE, L.; ENGLEZOS, V.; TORCHIO, F.; CRAVERO, F.; RÍO SEGADE, S.; RANTSIOU, K.; GIACOSA, S.; GAMBUTI, A.; GERBI, V.; COCOLIN, L. Alcohol reduction in red wines by technological and microbiological approaches: a comparative study. Australian Journal of Grape and Wine Research, Hoboken, v. 24, n. 1, p. 62-74, mai. 2018.

SEIDLITZ, H.; LACK, E.; LACKNER, H. Process for the reduction of the Alcohol content of alcoholic beverages. United States Patent, 5.034.238, 23 jul.1991. 5p.

SILVA, C.C.S. Avaliação do processo biotecnológico e determinação das condições de desalcoolização da bebida obtida por fermentação controlada de suco de maçã. 2004. 120f. Tese (Doutorado em Processos Biotecnológicos Industriais) - Departamento de Engenharia Química - Universidade Federal do Paraná, Curitiba, 2004.

SINGLETON, V.L.; ROSSI, J.A. Colorimetry of total phenolics with phosphomolybdicphosphotungstic acid reagents. American Journal of Enology and Viticulture, Davis, v. 16, n. 3, p. 144-158, 1965.

TAKÁCS, L.; VATAI, G.; KORÁNY, K. Production of alcohol free wine by pervaporation. Journal Food Engineering, Amsterdam, v. 78, n. 1, p. 118-125, 2007.

VAN CLEEF, E.H.C. B.; EZEQUIE, J.M.B.; GONÇALVES, J.S.; PASCOAL, L.A. F. Determinação da matéria seca das fezes de ovinos e da carne de peito de frango através do método tradicional e por liofilização. Revista Eletrónica de Veterinaria, Málaga, v. 11, n. 3, abr. 2010. Disponível em: https://bit.ly/2F4mxX1. Acesso em: 30 out. 2018.

VILANOVA, M.; GENISHEVA, Z.; GRAÑA, M.; OLIVEIRA, J. M. Determination of odorants in varietal wines from international grape cultivars (Vitis vinífera) Grown in NW Spain. South African Journal for Enology and Viticulture, Stellenbosch, v. 34, n. 2, p. 212-222, abr. 2013.

WINE INTELLIGENCE. Lower alcohol wine in the UK market. 2012, 59p.

WINE in the United Kingdom: Passport report. Euromonitor International: London, 2012, 28p. 